\title{
Genetic Characterization of Multidrug-Resistant Salmonella Typhimurium Carrying Mcr-1 in China
}

\section{Zhang Haoran}

Center for Disease Control and prevention of PLA

\section{Huang Yong}

Academy of Military Medical Sciences

\section{Liang Beibei}

Centers for Disease Control and Prevention of PLA

\section{Xie Jing}

Centers for Disease Control and Prevention of PLA

\section{Xiang Ying}

Centers for Disease Control and Prevention of PLA

\section{Du Xinying}

Centers for Disease Control and Prevention of PLA

\section{Yang Chaojie}

Center for disease control and prevention of PLA

\section{Liu Hongbo}

Centers for Disease Control and Prevention of PLA

\section{Liu HONGBo}

center for disease control and prevention of PLA

\section{Wang Hui}

Centers for Disease Control and Prevention of PLA

\section{Yang Mingjuan}

Centers for Disease Control and Prevention of PLA

\section{Wang Ligui}

Center For Disease control and prevention of PLA

\section{Hu Xiaofeng}

Centers for Disease Control and Prevention of PLA

Jin Lianqun

Center for Disease Control and Prevention of PLA

\section{Yuan Zhengquan}

Center for disease control and prevention of PLA

Qiu Shaofu ( $\nabla$ qiushf0613@hotmail.com )

CDC: Centers for Disease Control and Prevention of PLA 


\section{Research}

Keywords: Plasmid, Polymyxin, S. Typhimurium, Drug resistance

Posted Date: January 13th, 2021

DOI: https://doi.org/10.21203/rs.3.rs-143500/v1

License: (c) (i) This work is licensed under a Creative Commons Attribution 4.0 International License. Read Full License 
3 Running title: Genetic characterization of S. Typhimurium

5 Haoran Zhang ${ }^{1,2 \dagger}$, Yong Huang ${ }^{1 \dagger}$, Beibei Liang ${ }^{2 \dagger}$, Jing Xie ${ }^{2}$, Ying Xiang ${ }^{2}$, Xinying Du ${ }^{2}$, Chaojie Yang ${ }^{2}$,

6 Hongbo Liu², HONGBo Liu², Hui Wang², Mingjuan Yang², Ligui Wang ${ }^{2}$, Xiaofeng $\mathrm{Hu}^{2}$, Lianqun Jin², 7 Zhengquan Yuan ${ }^{2^{*}}$, Shaofu $\mathrm{Qiu}^{2}{ }^{*}$, and Hongbin Song ${ }^{2^{*}}$

8

${ }^{1}$ Academy of Military Medical Sciences, Beijing, China; address: 20 Fengtai Eastern Avenue, Beijing, 100071, China.

${ }^{2}$ Center for Disease Control and Prevention of PLA, Beijing, China; address: 20 Fengtai Eastern

"These authors contributed equally to this work.

\section{* Corresponding author}

Mailing address: Center for Disease Control and Prevention of PLA, Beijing, China; Beijing,

China; address: 20 Fengtai Eastern Avenue, Beijing, 100071, China; 
Background: With the rapid emergence of plasmid mediated polymyxin resistance gene mcr-1, multidrug-resistant Salmonella caused great troubles in clinical treatment and attracted extensive attention. Here we report multidrug-resistant Salmonella strains harboring mcr-1 in China, which are resistant to both polymyxin, traditional antibiotics and even clinically wide-used antibiotics.

Methods: We screened 1454 strains of Salmonella collected in our laboratory from 2006 to 2018 from 3 provinces or regions for $m c r-1$ by PCR. Antimicrobial susceptibility testing was determined. Plasmid conjugation assays were carried out to analysis the transferability of polymyxin resistance. Genetic polymorphism analysis of Salmonella was performed using the PFGE, and the plasmid profiles were characterized by S1-PFGE and southern blotting. The plasmids harboring $m c r-1$ were sequenced and compared.

Results: Eleven S. Typhimurium isolates harboring $m c r-1$ with polymyxin resistance (MICs $4 \mu \mathrm{g} / \mathrm{ml}$ ) were identified from intestinal infections and foods in China. All S. Typhimurium isolates were multidrug-resistant to traditional antibiotics and even clinically wide-used antibiotics. Three types of plasmids harboring $m c r-1$ were recovered (IncHI2, IncX4 and IncI2). Compared with the reference plasmid, IncX4 and IncI2 plasmids had extremely similar typical backbone, and contain only $m c r-1$ resistance gene. However, IncHI2 were the most diverse type of plasmid due to containing a large MDR region, including bla $a_{\mathrm{CTX}-\mathrm{M}}, o q x B, s u l, a p h, a a d A$ and $b l a_{\mathrm{TEM}}$. IncHI2 plasmids were observed to contain only one or no insertion sequence ISApl1 around mcr-1, without forming a circular intermediate.

Conclusion: With the horizontal transfer of different types of plasmids, $m c r-1$ is widely spread worldwide. These prevalent plasmids are responsible for resistance to polymyxin, traditional antibiotics and even clinically wide-used antibiotics resulting from transmission of $m c r-1$ and other resistance 
genes. Our studying emphasizes the necessity to jointly monitor its international epidemic and preemptive further upgrade.

Keywords: Plasmid; Polymyxin; S. Typhimurium; Drug resistance

\section{Introduction}

The emergence and evolution of multi-drug-resistant (MDR, resistance to three or more classes of antimicrobials) bacteria, including gram-negative bacteria, pose a growing threat to public health $[1,2]$.

Salmonella is one of the common pathogens causing bacterial intestinal infections and diarrhea in both developed and developing countries. In recent years, powerful fluoroquinolones and cephalosporins with a broad antibacterial spectrum have been developed as the preferred drugs in the clinical treatment of Salmonella infection [3]. In addition, azithromycin has been recommended for the clinical treatment of Salmonella infection due to its superior curative effect on intestinal bacteria in 2017. But with the increase in the MDR rate of Salmonella isolate, the drug sensitivity of fluoroquinolones, cephalosporins and azithromycin is also decreased, and the MDR Salmonella causing great troubles in clinical treatment have been reported in countries across six continents [4-6]. Polymyxin, as one of the colistin antibiotics, is currently the last line of defense the severe infection with MDR-Gram-negative bacteria [7]. A plasmid-mediated horizontally transferable colistin resistance gene, $m c r-1$, was first found in China in 2015, which plays an important role in the transmission of colistin resistant strains [8]. The colistin resistance gene $m c r-1$, was first detected from E. coli harboring a conjugative IncI2 plasmid in China. It encodes a phosphoethanolamine transferase which is modification of the lipid A.

The propagation of polymyxin resistance gene $m c r-1$ is closely related to these types of plasmids, which include IncHI1, IncHI2, IncI2, IncX4, IncF, IncFI, IncFII and IncP[3, 9]. It may provide the insight about potential of multiple drug resistance genes transfer between Salmonella species in 
the intestine. Here, our study was aimed to characterize the Salmonella enterica serovar Typhimurium (S. Typhimurium) harboring $m c r-1$ plasmid isolated from patients, pork and pork products.

\section{MATERIALS AND METHONDS}

\section{Bacterial Isolation, Serotyping, mcr-1 Gene Screening}

We conducted a retrospective mcr-1 study on 1454 strains of Salmonella collected in our laboratory from 2006 to 2018 from 3 provinces or regions (Shanghai 1046, Guangdong 209, Guangxi 199). These isolates were recovered from the stool samples of patients and market were strictly identified according to the national microbiological examination standard and stored in the glycerol tube at $-80^{\circ} \mathrm{C}$. Salmonella strains were identified through the use of biochemical test kit (API20E system Merrier; bioMérieux Vitek, Marcy-L'Etoile, France) according to the manufacturer's instructions. According to the Kauffmann-White scheme, Salmonella strains were serotyped on slides by a microtiter agglutination test for $\mathrm{O}$ and $\mathrm{H}$ antigens, strictly following the manufacturer's instructions (SSI, Copenhagen, Denmark). We screened all historical Salmonella strains for mcr-1 by PCR using the published primers according Liu study [8].

\section{Antimicrobial Susceptibility Testing}

Antimicrobial susceptibility testing was determined using broth microdilution in Sensititre Gram Negative AST Plates for Salmonella strains (Thermo Fisher Scientific, Inc., West Sussex, United Kingdom) including 14 different antimicrobials: ceftriaxone (CRO), tetracycline (TET), ceftiofur (XNL), cefoxitin (FOX), gentamicin (GEN), ampicillin (AMP), chloramphenicol (CHL), ciprofloxacin (CIP), trimethoprim/sulfamethoxazole (SXT), sulfisoxazole (FIS), nalidixic acid (NAL), streptomycin (STR), azithromycin (AZI), and amoxicillin/clavulanic acid 2:1 ratio (AUG2). The susceptibility to colistin is to use the dye WST (Dojindo Molecular Technologies, Inc., Japan) by a microbial viability 
assay kit. A reference strain of Escherichia coli ATCC 25922 strain was performed in the test as quality control [10].

\section{Plasmid conjugation assays}

Plasmid conjugation assays were determined using a standard E. coli j53 as the recipient, and the $m c r-1$ positive Salmonella strains as donors. The donor bacteria cultured overnight were mixed with the recipient bacteria in a ratio of $1: 3$, and were harvested, re-suspended in $80 \mu \mathrm{L}$. The mixture was incubated for mating at $37^{\circ} \mathrm{C}$ for $12-18 \mathrm{~h}$ in $5 \mathrm{ml} \mathrm{LB}$ liquid broth. Then a Muller-Hinton agar (BD Biosciences, San Jose, CA) plate containing $100 \mathrm{mg} / \mathrm{L}$ sodium azide and $2 \mathrm{mg} / \mathrm{L}$ polymyxin B was to a selective medium for E. coli j53 transconjugants. Putative transconjugants were confirmed by antimicrobial susceptibility testing and detection of $m c r-1$ with PCR.

\section{Pulsed-Field Gel Electrophoresis (PFGE), S1-PFGE and Southern}

\section{blotting}

Genomic polymorphism analysis of Salmonella strains was performed using the pulsed field gel electrophoresis (PFGE) after a slight modification of the pulseNet standardized PFGE protocol for Salmonella [11]. These isolates were digested with $\mathrm{XbaI}$ (Takara, Dalian, China) at $37^{\circ} \mathrm{C}$, and the salmonella enterica var. Braendrup H9812 strain was used as the reference. Electrophoresis running on

a CHEF MAPPER variable angle system (Bio-Rad, California, America) with the parameters set at 2.16s-63.8s for $19 \mathrm{~h}$ were performed following a previously methods [12]. The plasmid profiles were characterized by S1-PFGE. The endonuclease S1 nuclease (Takara, Dalian, China) was used to digest at $37^{\circ} \mathrm{C}$, and electrophoresis running set at $0.22 \mathrm{~s}-26.29 \mathrm{~s}$ for $15 \mathrm{~h}$. Following this, the southern blotting with digoxigenin-labelled $m c r-1$ probe sequences by using the published primers [8] was performed to step of membrane transfer, molecular hybridization, and probe detection following a previously 
reported method [13]. The images were captured by a Gel Doc 2000 system (Bio-Rad), and were imported into the BioNumerics software (v6.0) database for processing and further analysis.

\section{Whole Genome Sequencing and Bioinformatic Analysis}

The mcr-1 positive Salmonella strains preserved in our laboratory were sequenced based on the second-generation genome sequencing technique. The DNA was extracted from the overnight cultured strains according to the instructions of the QIAamp DNA Mini Kit (Qiagen, Hilden, Germany). The mate-pair library was constructed by nucleic acid protein analyzer Qsep100 to obtain DNA fragments (not less than 500bp, not more than 800bp) and sequenced by MiSeq platform sequencer. Sequence reads were assembled into draft continuous sequences (contigs) by Newbler [14] and NxTrim [15], and then splice it through GapFiller, Cytoscape. Complete plasmid genomes were annotated using the online annotation server RAST. Identification of insertion sequence (IS), plasmid replicons and resistance genes is performed by ISfinder (https://www-is.biotoul.fr/search.php), PlasmidFinder (https://cge.cbs.dtu.dk/services/PlasmidFinder/) and ResFinder [16], respectively. Multiple plasmids compared by Mauve, Brig and CLC Genomics Workbench. The circle figure of multiple plasmids for comparison were drawn by DNAploter, Circos et al [17].

\section{RESULT}

\section{Characterization of MDR salmonella harboring mcr-1}

A total of 1454 Salmonella strains were preserved in our laboratory from different samples collected from Shanghai (1046), Guangdong (209) and Guangxi (199), we found 11 strains of S. Typhimurium harboring $m c r-1$ gene. Among them, eight $m c r-1$ positive S. Typhimurium strains were identified from fecal samples of patients, and three mcr-1 positive $S$. Typhimurium strains were from pork and pork products. All eleven mcr-1 positive S. Typhimurium strains showed resistance to polymyxin (MICs 
$4 \mu \mathrm{g} / \mathrm{ml}$ ), and were resistance to multiple antimicrobial agents, including gentamicin, ampicillin, sulfamethoxazole, streptomycin, tetracycline and chloramphenicol. Moreover, six of eight strains isolated from patients were resistant to ceftriaxone and cefotaxime. Among three strains isolated from pork and pork products, one was resistant to ceftriaxone and cefotaxime, and another one was insensitive to azithromycin (Table 1).

\section{PFGE, plasmid profiling and southern blotting}

Eleven $m c r-1$ positive $S$. Typhimurium and four $m c r-1$ negative Salmonella were analyzed by PFGE.

Based on the PFGE results of fifteen isolates of Salmonella strains, four clusters were formed among the strains, which were divided according to about $85 \%$ homology. In Shanghai, the similarity of five mcr-1 positive Salmonella strains isolated from pork and humans in 2015 was above $95 \%$ with the mcr-1 negative Salmonella isolated from humans in 2012. The mor-1 negative Salmonella strains isolated from pork in 2014 had high homology with $m c r-1$ positive Salmonella isolated from human in 2015 and lower similarity with mcr-1 negative Salmonella isolated from pork in Guangdong. Further analysis of plasmid characteristics, S1-PFGE and southern blotting showed that all isolates carried different plasmids, of which two strains carried two plasmids, others carried one plasmid (Figure S1).

\section{Plasmid conjugation assays}

To assume the transfer ability of the $m c r-1$ positive plasmid, plasmid conjugation assays were performed using S. Typhimurium harbored $m c r-1$ gene as a donor and E. coli j53 as a recipient. Five (S49, S51, S52, S55, S56) of eleven recipients in this experiment tested positive for the $m c r-1$ gene via PCR amplification and sequence analysis. The MIC of colistin for the transconjugants (the E. coli j53 harboring $m c r-1$ gene) increased to $4 \mu \mathrm{g} / \mathrm{ml}$.

\section{Genetic characterization of plasmids harboring mcr-1}


We have obtained the plasmid sequence of eleven S. Typhimurium strains. Through the plasmid comparison on NCBI, we downloaded five highly homologous plasmids containing $m c r-1$ from Salmonella strains as a reference (at above $95 \%$ coverage and at above $99 \%$ identity). Through the analysis of plasmids, three IncX4, two IncI2 and six IncHI2 plasmids were identified to contain $m c r-1$ resistance genes among 11 strains. Three IncX4 plasmids were $33 \mathrm{~kb}$ in size, and were extremely similar to pNG14043 from Salmonella in Taiwan. These plasmids were typical IncX4 backbone which varied only by single nucleotide polymorphism (SNP) (at above 99\% homology). The insertion sequence IS26 was upstream of $m c r-1$ in our isolates, and IS15 was on these plasmids but absent around $m c r-1$. Those plasmids had only $m c r-1$ genes and no other identifiable resistance genes. (Fig. 2, Fig. 5). Two IncI2 plasmids were 50kb, and were similar to pHNSHP45 by E. coli strains from Shanghai in July, 2013. We found that these two IncI2 plasmids have a common plasmid backbone. However, compared with the two IncI2 plasmids, the region around $m c r-1$ were located in an inverted orientation. The insertion sequence ISApl1 and IS683 were missing in two IncI2 plasmids in this study by comparing with the reference plasmid sequence (Fig. 3, Fig. 5). Unlike the IncX4 and IncI2 types of plasmids, six IncHI2 plasmids were the most diverse type of plasmid due to containing a large MDR region. The MDR region is a region in the IncHI2 plasmid that contains a wide range of resistance genes, integrons and ISs. These IncHI2 plasmids that share a common backbone were $250 \mathrm{~kb}$, used pHNSHP45-2 as a reference plasmid with high homology. The MDR region of the reference plasmid contained a variety of resistance genes, including bla ${ }_{C T X-M}$, oqxA, oqxB, oqxR, sul, aph, aadA, dfrA, floR, aac, fosA, hph, intl. Some drug resistance genes and gene element can be observed in that region of the IncHI2 plasmids in our study, distinguishing them from the reference plasmid sequence (Table 1). Compared to the reference plasmid, part of drug-resistant genes in the MDR region has missed in 
our IncHI2 plasmids (pS49, pS54, pS70), including oqxA, oqxB, oqxR, sul, aadA; The drug-resistant gene $d f r A 12$ is missing in the MDR region of five IncHI2 plasmids except pS51 (Fig. 4). Among six IncHI2 plasmids, five IncHI2 plasmids were observed to contain the insertion sequence ISApl1 which was upstream of $m c r-1$, whereas another plasmid lacked ISApl1 around $m c r-1$ (Fig. 5).

\section{Discussion}

Salmonella is a common zoonotic pathogen that can cause bromatoxism and diarrhea of human beings $[5,10]$. For some special populations, antibiotic therapy is necessary for Salmonella infection. According to the Salmonella monitoring report, the MDR of Salmonella increased 40 percent in the last decade of 20th century [18, 19]. Among the many serotypes of Salmonella, the MDR of $S$. Typhimurium, has drawn global concern in particular. However, the effects of traditional antibiotics and even clinically wide-used antibiotics to S. Typhimurium have been reduced, which include fluoroquinolones, cephalosporins and even azithromycin [20].

Polymyxin has been used as the ultimate means of combating multidrug-resistant Gram-negative bacterial infections [21]. Since 2015, polymyxin has been listed to be one of the crucially important antibiotics by WHO, while it has been approved for treatment of bacterial infections since 2017 [22]. But plasmid mediated polymyxin resistant strains with $m c r-1$ positive were first reported in China in 2015[8]. Eleven S. Typhimurium strains harbored $m c r-1$ gene were found in a retrospective study from 1454 strains in our laboratory. Under the analysis, we found that multi-drug resistance existed in all these eleven mor-1 positive S. Typhimurium strains. These eleven polymyxin resistant S. Typhimurium strains from different sources have multi-drug resistance, resisting nalidixic acid, gentamicin, ampicillin, sulfisoxazole, streptomycin, tetracycline, and chloramphenicol, of which eight were from the stools of patients with diarrhea and three were from pork and pork products. Even the 
third-generation antibiotics such as cephalosporins and azithromycin, which are clinically common-used to treat Salmonella infections, have become noneffective. It is important to note that all eight patients infected by mcr-1 positive MDR $S$. Typhimurium are all children under 4 years of age (Table S1). This finding is consistent with the results reported by Luo et al [23], which most of the Salmonella infected people are children under 5 years old and patients with low immunity. At the same time, the results of PFGE prompted us, five strains of polymyxin resistant S. Typhimurium isolated from children under 4 years and pork in Shanghai in 2015 had high homology. These isolates were highly homologous with the $m c r-1$ negative Salmonella in the same region in 2012 and were not in the same cluster with the strains from Guangdong and Fujian. Both the above results and plasmid conjugation assays suggest that the $m c r-1$ gene can be transmitted between local strains by horizontal transfer of plasmids, and these plasmids may simultaneously carry a variety of different resistance genes, resulting in an increase of MDR S. Typhimurium strains. The emergence of $m c r-1$ positive MDR S. Typhimurium strains may lead to serious consequences such as the increasing difficulty of future treatment, therapeutic failure and even death $[24,25]$. In the Salmonella strains, plasmids play a vital role in the acquisition of drug resistance caused by drug resistance genes [26]. The $m c r-1$ and other resistance genes are usually located on the same or different plasmids. These genes are widely spread in animals, environment and food in many countries and regions around the world through horizontal transfer of plasmids [27]. It is demonstrated that $m c r-1$ and other resistance genes with the spread of Salmonella strains have potential to transfer from food product animal strains to human strains [28, 29]. During plasmid transfer, the $m c r-1$ positive plasmids display significant diversity in terms of antibiotic resistance patterns, incompatibility groups, and genetic contents [3]. IncI2 and IncX4 plasmids which promote Salmonella resistance are the two main 
types of plasmids spreading globally [14]. In the study, eleven strains of S. Typhimurium found that the plasmids containing $m c r-1$ were mainly located in IncI2, IncX4 and IncHI2. According to reports in Enterobacteriaceae [30], IncHI2 plasmid (216-280kb), containing a multi-drug resistance region, is the fifth most common plasmid family, and is one of the main plasmid groups carrying $m c r-1$ gene variants. We observed the coexistence of plasmids containing $m c r-1$ and multiple drug resistance genes in this work. The reduced sensitivity to multiple antibiotics is attributed to the fact that $m c r-1$ positive $S$. Typhimurium also coharbored $o q x B, b l a_{\mathrm{TEM}}$ and $b l a_{\mathrm{CTX}}$ resistance genes. These genes are co-located in drug-resistant plasmids, resulting in decreased antibiotic susceptibility to polymyxin, quinolones and the third-generation cephalosporins. The transfer of IncHI2 plasmids carrying multiple drug resistance genes between bacteria caused the rapid spread of antibiotic resistance in a certain area. MDR IncHI2 plasmids containing $m c r-1$ are widely distributed in human pathogens, and are the most efficient vectors for the transmission of $m c r-1$ and other drug-resistant genes. The worrying assumption is that MDR IncHI2 plasmids in the dissemination of $m c r-1$ and other drug-resistant genes cause the change of Salmonella resistance phenotype and spread within a short period of time.

The insertion sequence (IS) family in the bacterial genome, a widely variable DNA sequence in nature, is also one of the important ways for the transmission of resistance genes between bacterial pathogens [12]. The eleven plasmids are divided into different patterns according to the presence or absence of IS elements and the relationship with $m c r-1$ sites. The different types and positions of IS sequences in these plasmids are both exposed the potential threat presented by Salmonella strains carrying $m c r-1$ plasmids via their role in transmitting the polymyxin resistance gene. ISApl1 possesses significant role in the transmission of $m c r-1$. In our study, ISApl1 element is closely related to the IncHI2 type plasmid. This suggests that under the pressure of various antibiotics in the natural environment, strains may 
obtain $m c r-1$ and other resistance elements distributed in different plasmids through a circular intermediate with the aid of ISApl1 [12]. The stark truth is that the $m c r-1$ is general associated with the $m c r-1$ gene cassettes, designated as Tn6330. Tn6330 containing ISApl1-mcr-1-orf- ISApl1 structure can insert mcr-1 into different types of plasmids by forming a circular intermediate. In our research, five of six IncHI2 plasmids were observed to contain the insertion sequence ISApl1 which was upstream of $m c r-1$, whereas another plasmid lacked ISApl1 around $m c r-1$. The $m c r-1$ translocation could be mediated the insertion of the $m c r-1$ gene into the IncHI2 plasmid backbone, and possibly other plasmids through $m c r-1$ gene cassettes. Indeed, these two types of insertion sequences in our IncHI2 plasmids cannot detect Tn6330. It should be noted that the plasmids like these two types are more stable in Salmonella strains.

\section{Conclusion}

This study describes in detail the genetic characteristics of $m c r$-1-postive $S$. Typhimurium strains from patients, pork and pork products in China. Three types of plasmids sharing a common plasmid backbone have their own characteristics regarding IS and resistance genes. These prevalent plasmids are responsible for resistance to polymyxin, traditional antibiotics and even clinically wide-used antibiotics resulting from transmission of mcr-1 and other resistance genes. Therefore, these findings emphasize the necessity of assessing the potential of mcr-1 to spread and jointly monitoring its international epidemic and preemptive further upgrade.

\section{Accession number}

The complete sequences of plasmids in this study have been deposited in GenBank under accession numbers MW264504- MW264516, respectively.

\section{Acknowledgements}

The present study was funded by grants from the National Key R\&D Program of China (no. 


\section{Disclosure}

The authors report no conflicts of interest in this work.

\section{References}

1. Jain P, Chowdhury G, Samajpati S, Basak S, Ganai A, Samanta S et al:: Characterization of non-typhoidal Salmonella isolates from children with acute gastroenteritis, Kolkata, India, during 2000-2016. 2020, 51(2):613-627.doi:10.1007/s42770-019-00213-z.

2. Tang B, Chang J, Zhang L, Liu L, Xia X, Hassan BH et al:: Carriage of Distinct mcr-1-Harboring Plasmids by Unusual Serotypes of Salmonella. Adv Biosyst 2020, 4(3):e1900219.doi:10.1002/adbi.201900219.

3. Touati A, Mairi A: Plasmid-Determined Colistin Resistance in the North African Countries: A Systematic Review. Microb Drug Resist 2020.doi:10.1089/mdr.2019.0471.

4. Wang Y, Xu C, Zhang R, Chen Y, Shen Y, Hu F et al:: Changes in colistin resistance and mcr-1 abundance in Escherichia coli of animal and human origins following the ban of colistin-positive additives in China: an epidemiological comparative study. The Lancet Infectious Diseases 2020.doi:10.1016/s1473-3099(20)30149-3.

5. Ling Z, Yin W, Shen Z, Wang Y, Shen J, Walsh TR: Epidemiology of mobile colistin resistance genes mcr-1 to mcr-9. J Antimicrob Chemother 2020.doi:10.1093/jac/dkaa205.

6. Anyanwu MU, Jaja IF, Nwobi OC: Occurrence and Characteristics of Mobile Colistin Resistance (mcr) Gene-Containing Isolates from the Environment: A Review. Int $J$ Environ Res Public Health 2020, 17(3).doi:10.3390/ijerph17031028.

7. Lu X, Zeng M, Xu J, Zhou H, Gu B, Li Z et al.: Epidemiologic and genomic insights on mcr-1-harbouring Salmonella from diarrhoeal outpatients in Shanghai, China, 2006-2016. EBioMedicine 2019, 42:133-144.doi:10.1016/j.ebiom.2019.03.006.

8. Liu Y, Wang Y, Walsh T, Yi L, Zhang R, Spencer J et al.: Emergence of plasmid-mediated colistin resistance mechanism MCR-1 in animals and human beings in China: a microbiological and molecular biological study. 2016, 16(2):161-168.doi:10.1016/s1473-3099(15)00424-7.

9. Zurfluh K, Nuesch-Inderbinen M, Klumpp J, Poirel L, Nordmann P, Stephan R: Key features of mcr-1-bearing plasmids from Escherichia coli isolated from humans and food. Antimicrob Resist Infect Control 2017, 6:91.doi:10.1186/s13756-017-0250-8.

10. Wang J, Li Y, Xu X, Liang B, Wu F, Yang X et al.: Antimicrobial Resistance of Salmonella enterica Serovar Typhimurium in Shanghai, China. Front Microbiol 2017, 8:510.doi:10.3389/fmicb.2017.00510.

11. Ribot E, Fair M, Gautom R, Cameron D, Hunter S, Swaminathan B et al.: Standardization of 
pulsed-field gel electrophoresis protocols for the subtyping of Escherichia coli 0157:H7, Salmonella, and Shigella for PulseNet. 2006, 3(1):59-67.doi:10.1089/fpd.2006.3.59.

12. Liu H, Zhu B, Liang B, Xu X, Qiu S, Jia L et al.: A Novel mcr-1 Variant Carried by an IncI2-Type Plasmid Identified From a Multidrug Resistant Enterotoxigenic Escherichia coli. Front Microbiol 2018, 9:815.doi:10.3389/fmicb.2018.00815.

13. Zou D, Huang $\mathrm{Y}$, Zhao X, Liu W, Dong D, Li $\mathrm{H}$ et al.: A novel New Delhi metallo- $\beta$-lactamase variant, NDM-14, isolated in a Chinese Hospital possesses increased enzymatic activity against carbapenems. 2015, 59(4):2450-2453.doi:10.1128/aac.05168-14.

14. Arredondo-Alonso S, Rogers M, Braat J, Verschuuren T, Top J, Corander J et al.: mlplasmids: a user-friendly tool to predict plasmid- and chromosome-derived sequences for single species. 2018, 4(11).doi:10.1099/mgen.0.000224.

15. O'Connell J, Schulz-Trieglaff O, Carlson E, Hims M, Gormley N, Cox AJB: NxTrim: optimized trimming of Illumina mate pair reads. 2015, 31(12):2035-2037.doi:10.1093/bioinformatics/btv057.

16. Bortolaia V, Kaas R, Ruppe E, Roberts M, Schwarz S, Cattoir V et al: : ResFinder 4.0 for predictions of phenotypes from genotypes. 2020.doi:10.1093/jac/dkaa345.

17. Roberts A, Chandler M, Courvalin P, Guédon G, Mullany P, Pembroke T et al.: Revised nomenclature for transposable genetic elements. 2008, 60(3):167-173.doi:10.1016/j.plasmid.2008.08.001.

18. Elbediwi M, Pan H, Biswas S, Li Y, Yue M: Emerging colistin resistance in Salmonella enterica serovar Newport isolates from human infections. Emerg Microbes Infect 2020, 9(1):535-538.doi:10.1080/22221751.2020.1733439.

19. Osei Sekyere J, Maningi NE, Modipane L, Mbelle NM: Emergence of mcr-9.1 in Extended-Spectrum-beta-Lactamase-Producing Clinical Enterobacteriaceae in Pretoria, South Africa: Global Evolutionary Phylogenomics, Resistome, and Mobilome. mSystems 2020, 5(3).doi:10.1128/mSystems.00148-20.

20. Huang H, Dong N, Shu L, Lu J, Sun Q, Chan E et al:: mcrColistin-resistance gene in clinical carbapenem-resistant strains in China, 2014-2019. 2020, 9(1):237-245.doi:10.1080/22221751.2020.1717380.

21. Bai F, Li X, Niu B, Zhang Z, Malakar PK, Liu H et al:: A mcr-1-Carrying Conjugative IncX4 Plasmid in Colistin-Resistant Escherichia coli ST278 Strain Isolated From Dairy Cow Feces in Shanghai, China. Front Microbiol 2018, 9:2833.doi:10.3389/fmicb.2018.02833.

22. Li R, Xie M, Zhang J, Yang Z, Liu L, Liu X et al.: Genetic characterization of mcr-1-bearing plasmids to depict molecular mechanisms underlying dissemination of the colistin resistance determinant. J Antimicrob Chemother 2017, 72(2):393-401.doi:10.1093/jac/dkw411.

23. Luo Q, Wan F, Yu X, Zheng B, Chen Y, Gong C et al.: MDR Salmonella enterica serovar Typhimurium ST34 carrying mcr-1 isolated from cases of bloodstream and intestinal infection in children in China. $J$ Antimicrob Chemother 2020, 75(1):92-95.doi:10.1093/jac/dkz415.

24. Folster J, Grass J, Bicknese A, Taylor J, Friedman C, Whichard JJMdr: Characterization of Resistance Genes and Plasmids from Outbreaks and Illness Clusters Caused by Salmonella Resistant to Ceftriaxone in the United States, 2011-2012. 2017, 
23(2):188-193.doi:10.1089/mdr.2016.0080.

25. Wang Y, Cao C, Alali W, Cui S, Li F, Zhu J et al:: Distribution and Antimicrobial Susceptibility of Foodborne Salmonella Serovars in Eight Provinces in China from 2007 29. Pornsukarom S, van Vliet AHM, Thakur S: Whole genome sequencing analysis of multiple and animal source foods in China. Int $J$ Food Microbiol 2020, 335:108859.doi:10.1016/j.ijfoodmicro.2020.108859.

27. Huang H, Dong N, Shu L, Lu J, Sun Q, Chan EW et al.: Colistin-resistance gene mor in clinical carbapenem-resistant Enterobacteriaceae strains in China, 2014-2019. Emerg Microbes Infect 2020, 9(1):237-245.doi:10.1080/22221751.2020.1717380.

28. Ma Y, Xu X, Gao Y, Zhan Z, Xu C, Qu X et al.: Antimicrobial resistance and molecular characterization of Salmonella enterica serovar Corvallis isolated from human patients Salmonella serovars provides insights into phylogenetic relatedness, antimicrobial resistance, and virulence markers across humans, food animals and agriculture environmental sources. BMC Genomics 2018, 19(1):801.doi:10.1186/s12864-018-5137-4.

30. Zingali T, Chapman TA, Webster J, Roy Chowdhury P, Djordjevic SP: Genomic Characterisation of a Multiple Drug Resistant IncHI2 ST4 Plasmid in Escherichia coli ST744 in Australia. Microorganisms 2020, 8(6).doi:10.3390/microorganisms8060896. 
Table 1 Characteristic of eleven mcr-1-positive MDR S. Typhimurium

\begin{tabular}{|c|c|c|c|c|c|c|}
\hline \multirow[b]{2}{*}{ Strain no. } & \multirow[b]{2}{*}{ Antibiogram } & \multicolumn{5}{|c|}{ Results of sequencing for eleven $m c r$ - 1 -positive plasmids } \\
\hline & & $\begin{array}{c}\text { Plasmid } \\
\text { name }\end{array}$ & $\begin{array}{l}\text { Size of } m c r-1 \\
\text { plasmid(b) }\end{array}$ & $\begin{array}{c}\text { Type of } m c r-1 \\
\text { plasmid }\end{array}$ & Drug-resistant gene & IS Types \\
\hline S49 & $\begin{array}{c}\text { CRO,EFT,AMP,GE } \\
\text { N,SM,SX,C,COL }\end{array}$ & pS49 & 222291 & IncHI2 & $\begin{array}{c}m c r-1, b l a_{C T X-M}, a a c \\
\text { floR, aph, fosA }\end{array}$ & ISApIl \\
\hline S51 & $\begin{array}{c}\text { CRO,EFT,AMP,GE } \\
\text { N,SM,SX,SXT,C,T } \\
\text { E,COL }\end{array}$ & pS51 & 249475 & IncHI2 & $\begin{array}{l}\text { mcr-1, bla } a_{C T X-M}, s u l, \\
\text { oqxA, oqxR, dfrA, } \\
\text { floR, oqxB, aadA, } \\
\text { aph, aac, fosA, cml }\end{array}$ & ISApIl \\
\hline S52 & $\begin{array}{c}\text { CRO,EFT,AMP,GE } \\
\text { N,SM,SX,C,TE,CO } \\
\text { L }\end{array}$ & pS52 & 249043 & IncHI2 & $\begin{array}{c}m c r-1, b l a_{C T X-M}, \\
\text { oqxB, sul, aph, aadA, } \\
\text { fosA, floR, aac, cml, } \\
\text { oqxR }\end{array}$ & ISApIl \\
\hline S53 & $\begin{array}{l}\text { CRO,EFT,AMP,GE } \\
\text { N,SM,SX,C,COL }\end{array}$ & pS53 & 228926 & IncHI2 & $\begin{array}{c}\text { mcr-1, bla } a_{C T X-M}, \\
\text { oqxA, sul, oqxR, } \\
\text { aadA }\end{array}$ & ISApIl \\
\hline S54 & $\begin{array}{c}\text { CRO,EFT,AMP,GE } \\
\text { N,SM,SX,C,COL }\end{array}$ & pS54 & 222880 & IncHI2 & $\begin{array}{c}m c r-1, \text { bla } \\
\text { sul }, \text { aph-м, }, \text { floR, fos } A\end{array}$ & ISApIl \\
\hline S55 & $\begin{array}{l}\text { FOX,AMC,CRO,E } \\
\text { FT,AMP,SM,SX,S } \\
\text { XT,AZ,C,TE,COL }\end{array}$ & pS55 & 59233 & IncI2 & $m c r-1$ & none \\
\hline S56 & $\begin{array}{c}\text { COL } \\
\text { AMP,NAL,GEN,S }\end{array}$ & pS56 & 60454 & IncI2 & $m c r-1$ & none \\
\hline S60 & $\begin{array}{c}\text { M,SX,SXT,C,TE,C } \\
\text { OL }\end{array}$ & pS60 & 33308 & IncX4 & $m c r-1$ & IS15, IS26 \\
\hline S67 & $\begin{array}{c}\text { AMP,NAL,SM,SX, } \\
\text { TE,COL }\end{array}$ & pS67 & 33308 & IncX4 & $m c r-1$ & IS15, IS26 \\
\hline S69 & $\begin{array}{l}\text { AMP,NAL,GEN,S } \\
\text { X,SXT,C,TE,COL }\end{array}$ & pS69 & 33308 & IncX4 & $m c r-1$ & IS15, IS26 \\
\hline S70 & $\begin{array}{c}\text { CRO,EFT,AMP,GE } \\
\text { N,SM,SX,C,TE,CO } \\
\text { L }\end{array}$ & pS70 & 223256 & IncHI2 & $\begin{array}{c}m c r-1, b_{\text {CTX-M }}, \text { aac } \\
\text { sul, aph, floR }, \text { fosA }\end{array}$ & ISApIl \\
\hline
\end{tabular}




\section{Figure legends}

406

407 Fig1. Pulsed field gel electrophoresis (PFGE) of the fifteen Salmonella isolates in 2015. (A) Results of

PFGE profiles of Salmonella were clustered. (B) The key, species, origin, source and isolation year of the fifteen Salmonella are shown.

Fig2. Sequencing alignment of $m c r-1$ harbouring IncX4 plasmids. The $m c r-1$ harbouring plasmid pNG14043 with GenBank no.KY120364 which was isolated from S. Typhimurium in Taiwan was used as refrence plasmid (black circle). The outmost circle in red arrows denots the annotations of refrence plasmid. The figure shows the extremly high degree of homology of the four $m c r-1$ harbouring IncX4 plasmids. Detailed information of $m c r-1$ location of plasmids is provided in fugure5.

Fig3. Sequencing alignment of $m c r-1$ harbouring IncI2 plasmids. The first $m c r-1$ harbouring plasmid, pHNSHP45 with GenBank no.KP347127 which was isolated from E. coli strains from Shanghai in July, 2013 was used as refrence plasmid (black circle). The outmost circle in red arrows denots the annotations of refrence plasmid. The IS683 and ISApl1 are absent in two IncI2 plasmids in this study.

Detailed information of $m c r-1$ location of plasmids is provided in fugure5. plasmids, pSH51, pS52 and pS53 exhibit sequence with the refrence sequence, others are low sequence homology. An MDR region is exhibieted in the five IncHI2 plasmids. Detailed information of $m c r-1$ 
location of plasmids is provided in fugure5.

432 Fig5. Genetic context of eleven plasmids surrouding the $m c r-1$ gene. In CDS, red and blue arrows represent $m c r-1$ and IS respectively, black arrows present plasmid bone. The light blue and orange shaded regions indicate genetic regions that show the direct and reverse nucleotide ideatityhomology between different segments( $>99 \%)$. 


\section{Figures}

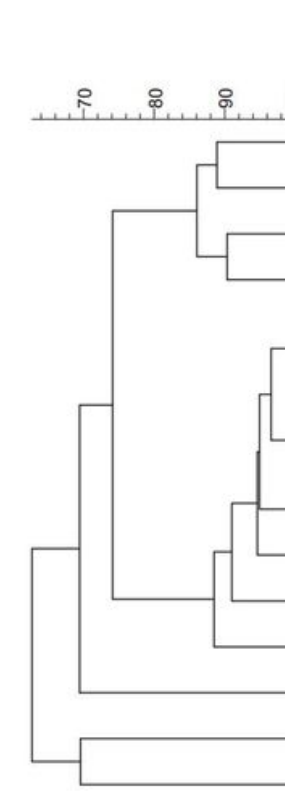

\section{PFGE-Xbal}

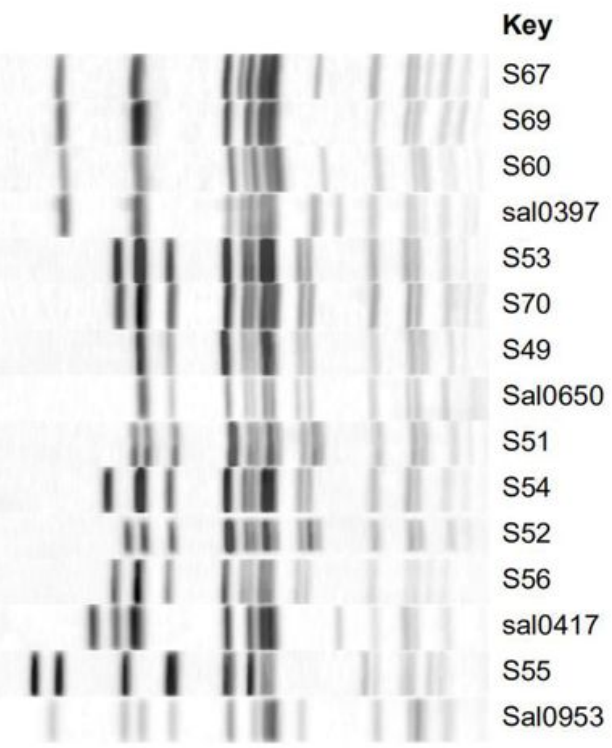

B

Species
Typhimurium
Typhimurium
Typhimurium
Typhimurium
Typhimurium
Typhimurium
Typhimurium
Typhimurium
Typhimurium
Typhimurium
Typhimurium
Typhimurium
Typhimurium
Typhimurium
Typhimurium

$\begin{array}{ll}\text { Source } & \text { Year } \\ \text { Pork } & 2015 \\ \text { Pork } & 2015 \\ \text { Human } & 2015 \\ \text { Pork } & 2014 \\ \text { Human } & 2015 \\ \text { Pork } & 2015 \\ \text { Human } & 2015 \\ \text { Human } & 2012 \\ \text { Human } & 2015 \\ \text { Human } & 2015 \\ \text { Human } & 2015 \\ \text { Human } & 2015 \\ \text { Pork } & 2013 \\ \text { Human } & 2015 \\ \text { Human } & 2015\end{array}$

\section{Figure 1}

Pulsed field gel electrophoresis (PFGE) of the fifteen Salmonella isolates in 2015. (A) Results of PFGE profiles of Salmonella were clustered. (B) The key, species, origin, source and isolation year of the fifteen Salmonella are shown. 


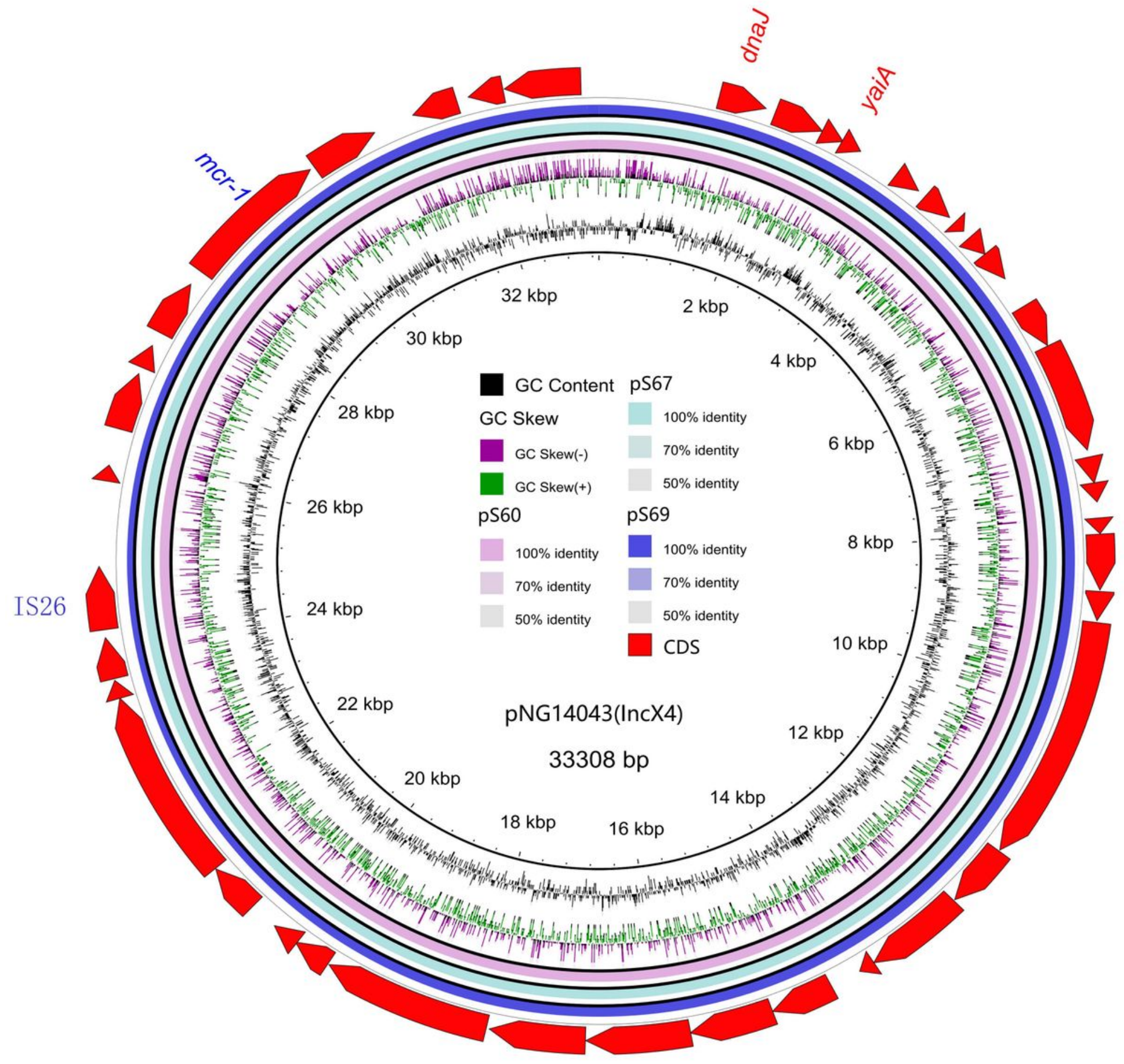

Figure 2

Sequen cing alignme nt of mcr 1 harbouring IncX4 plasmids. The mcr 1 harbouring plasmid pNG14043 with GenBank no. KY120364 which was isolated from S. Typhimurium in Taiwan was used as refrence plasmid (black circle). The outmost circle in red arrows denots the anno tations of $r$ efrence $\mathrm{pl}$ asmid. The figure shows the extremly high degree of homology of the four mcr 1 harbouring IncX4 plasmids. Detailed information of mcr 1 location of plasmids is provided in fugure 5 . 


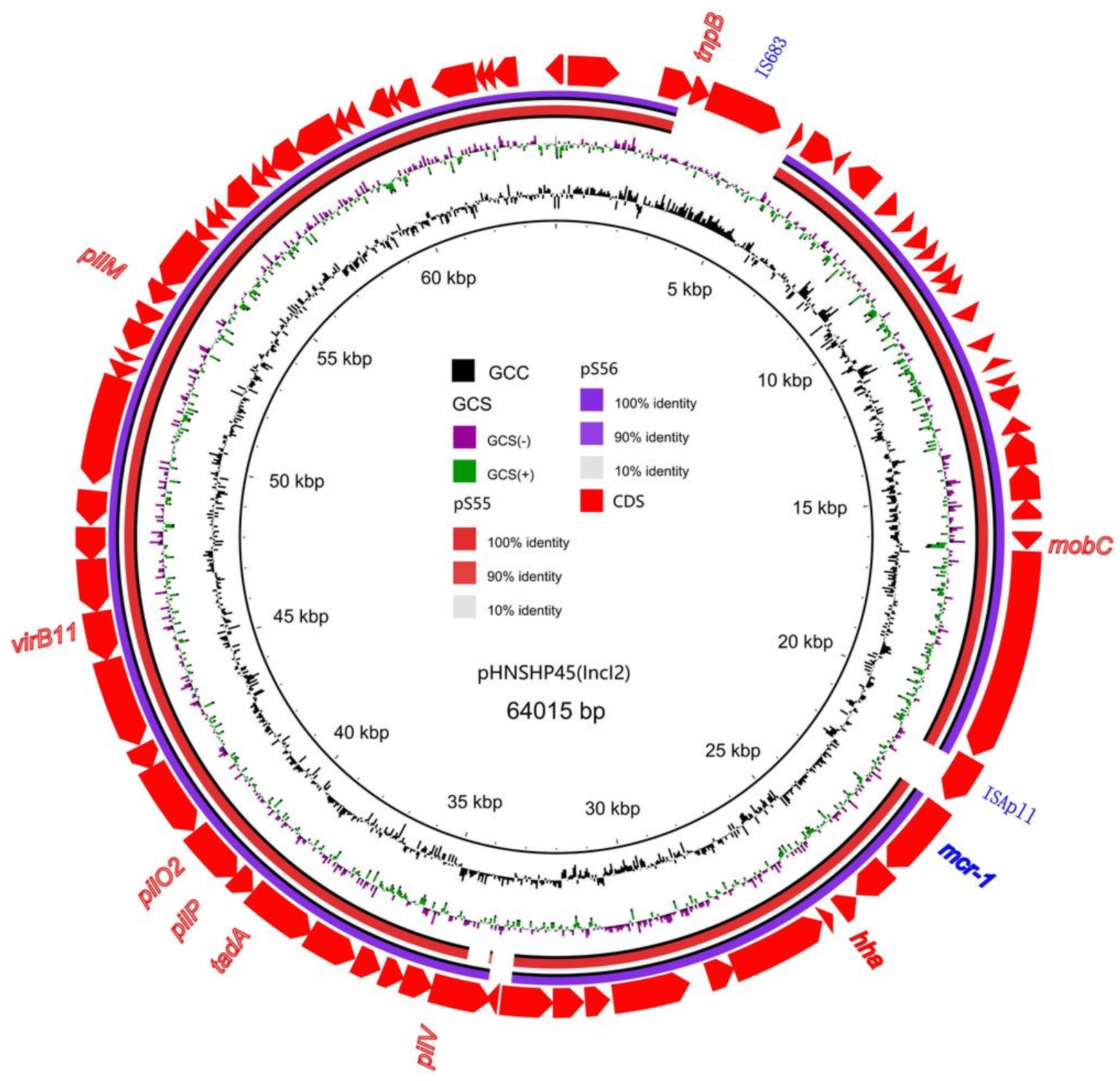

Figure 3

Sequencing alignment of mcr 1 harbouring Incl2 plasmids. The first mcr 1 harbouring plasmid, pHNSHP45 with GenBank no. KP347127 which was isolated from E. coli strains from Shanghai in July, 2013 was used as refrence plasmid (black circle). The outmost circle in red arrows denots the annotations of ref rence plasm id. The IS 683 and ISApl1 are absent in two Incl2 plasmids in this study. Detailed information of mcr 1 location of plasmids is provided in fugure5. 


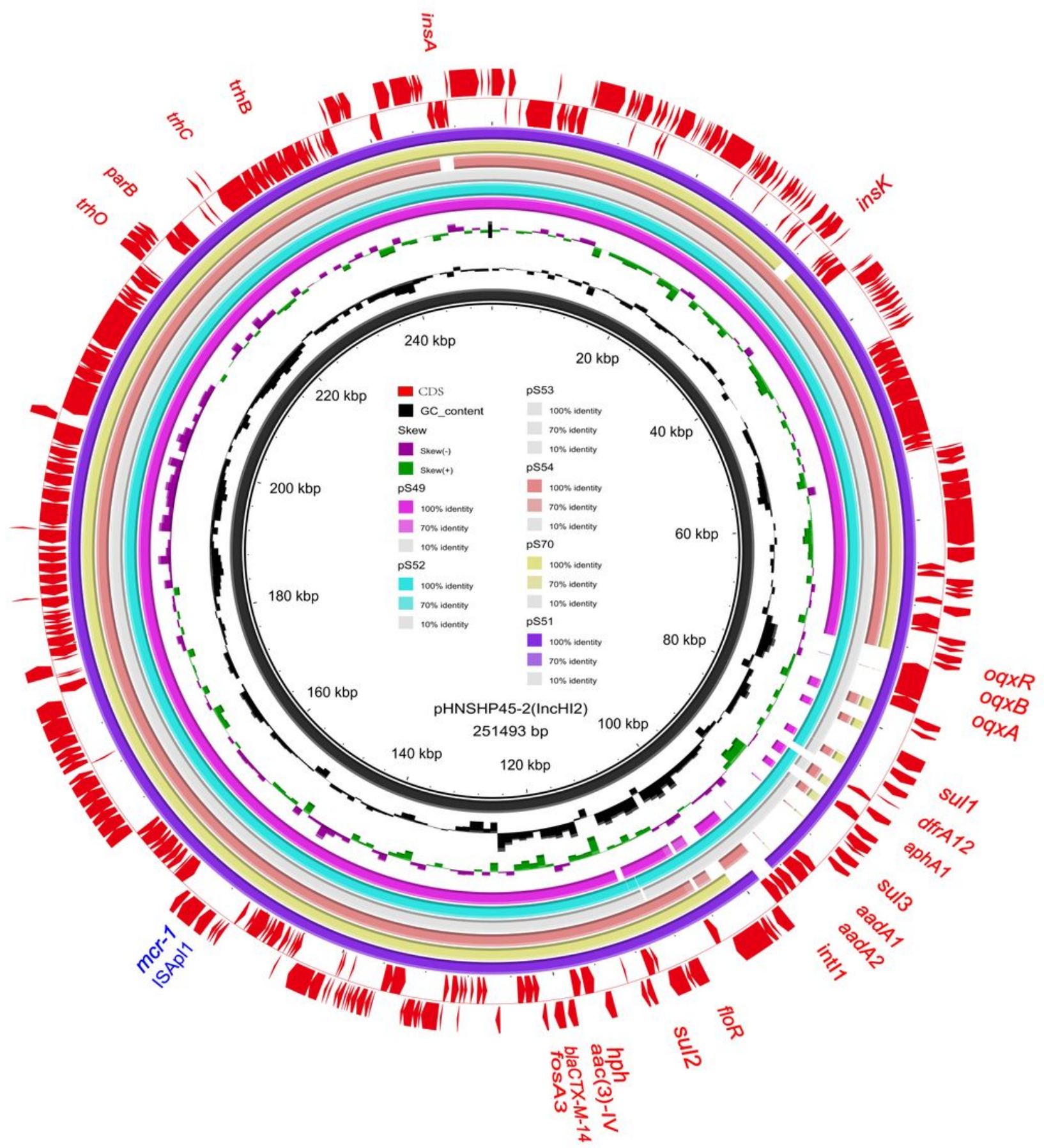

Figure 4

Sequencing alignment of mcr 1 harbouring IncHI2 plasmids. pHNSHP45 2 with GenBank no. KU34 1381 which was isola ted from E. coli strains was used as refrence plasmid (black circle). The outmost circle in red arrows denots the annotations of refrence plasmid. Among the six IncHI2 plasmids, pSH 51, pS 52 and pS53 exhibit sequence with the refrence se quence, ot hers are I ow sequence homology. An MDR region is exhibieted in the five IncHI2 plasmids. Detailed information of mcr 1 location of plasmids is provided in fugure5. 


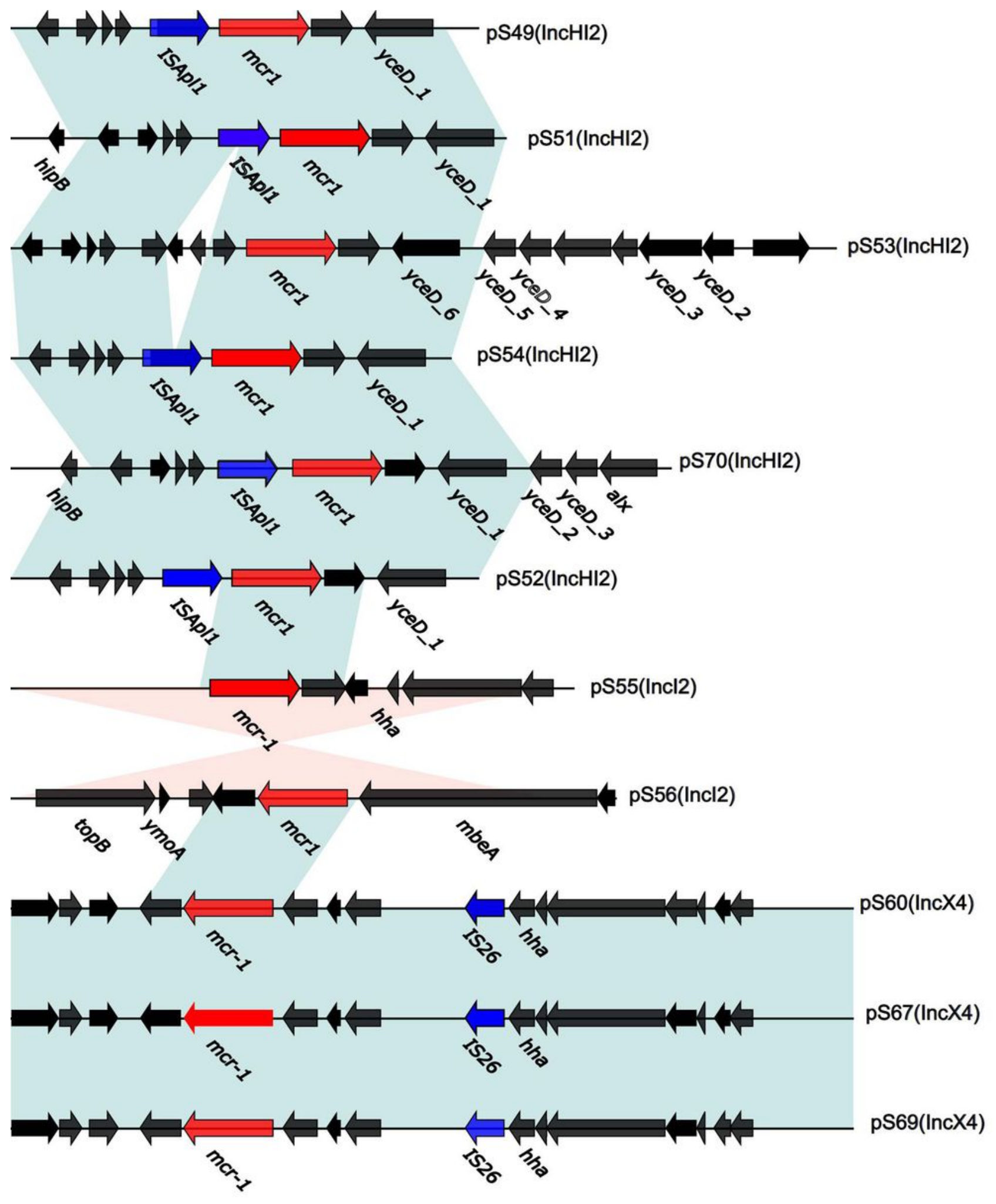

Figure 5

Genetic context of eleven plasmids surrouding the mcr 1 gene. In CDS, red a nd blue a rrows repr esent mcr 1 and IS respectively, black arrows present plasmid bone. The light blue and orange shaded regions indicate genetic regions that show the direct and reverse nucleotide ideatityhomology between different segments( $>99 \%)$ 


\section{Supplementary Files}

This is a list of supplementary files associated with this preprint. Click to download.

- FigS1.jpg

- AdditionalFiles.doc 